\title{
Teleradiology Image Transmission System: Diagnostic Accuracy at Three Matrix Sizes for Various Types of Images
}

\author{
Carrolf R. Markivee and Jeffrey L. Chenoweth
}

\begin{abstract}
The interpretability of transmitted digitized radiographic images is an important factor in the operation and use of teleradiology systems. Matrix size may influence diagnostic accuracy of the interpretation. Commercially available equipment has certain built-in image enhancement controls that may increase the interpretability of the image received. Evaluation of the effect of varying the matrix size and other factors on diagnostic accuracy are described. (c) 1990 by W.B. Saunders Company.
\end{abstract}

KEY WORDS: teleradiology, image, video.

$\mathbf{T}$ ELERADIOLOGY may be defined as the radiologic interpretation of digitized radiographic images following their transmission from a remote site. In current practice with commercially available equipment, the original image, a conventional radiograph, may be acquired by a video camera, or a laser scanner, then digitized and stored on a hard disk of a desktop microcomputer that has circuit boards and software capable of image enhancement and transmission.

Acquisition may be performed using storage phosphor media in place of $x$-ray film, with laser scan conversion of the inherent image, and also by direct input of digital data from computed tomography (CT) scanners, magnetic resonance (MR) scanners, and digital ultrasound equipment.

The images are transmitted via telephone lines or satellite transmission to another similarly configured microcomputer, stored on its hard disk, and interpreted by the radiologist when convenient. Transmission time per image varies from 30 seconds to 3 minutes.

The previous work of Gitlin ${ }^{1}$ and Harrington ${ }^{2}$ has shown that greater image matrix size results in increased diagnostic accuracy of the transmitted image. The disadvantages of greater matrix size are higher equipment cost, and longer transmission time. Doubling the matrix size requires

From the Department of Radiology, St Louis University Medical Center, and John Cochran Veterans Affairs Medical Center, St Louis, MO.

Address reprint requests to Carroll $R$. Markivee, $M D, S t$

Louis University Medical Center, St Louis, MO63106.

(C) 1990 by W.B. Saunders Company.

0897-1889/90/0303-0013\$03.00/0 four times as much disk storage and increases transmission time by four. Data compression may be increased, for example, 10 to 1 instead of 2 to 1 . Transmission time will be decreased, but the reconstructed image at the receive module shows perceptible image degradation.

\section{MATERIALS AND METHODS}

We used a commercially available image transmission system (Ektascan, Eastman Kodak Co, Rochester, NY) which acquires images using a vidicon camera with a macrozoom lens mounted above a conventional viewbox, and interfaced to an IBM (Boca Raton, FL) PC AT microcomputer. Optical magnification of the image prior to acquisition is possible up to $4 \times$. Acquisition from radiographs as large as 14 in by 17 in produce a $512 \times 480$ matrix on a special image display monitor ( 32 lines are lost during the retrace).

\section{Hardware Configuration}

The basic hardware configuration was described previously. ${ }^{3}$ The display monitor has a panel of image manipulation controls. These are (1) window width/level control; (2) histogram equalization; (3) reverse video; (4) area of interest; (5) grid overlay; and (6) zoom. The window width/level function allows continuous reduction of the display of gray levels from 255 to 10 levels and centering of the window at any level from 10 to 245 . The histogram equalization function reassigns pixel gray level values linearly, in an area marked by cursors by the user and displays the full range of shades of gray in that area. Reverse video reverses black and white. Area of interest may be marked for subsequent transmission. The grid overlay identifies relative positions on the image by column and row. Electronic zoom magnifies the stored image by spreading the pixels farther apart. Two hundred and fifty six shades of gray are provided on the Ektascan image display monitor.

\section{Radiographic Image Database}

A total of 116 images were digitized, transmitted, and evaluated. Images included 57 coarse detail and 59 fine detail images. The database of coarse detail images consisted of 8 radiographs with large abnormalities, together with $41 \mathrm{CTs}$ and 8 ultrasound images transmitted 12-on-1, 4-on-1, and 1-on-1 (Table 1).

The database of fine detail images consisted of 42 chest and 17 bone radiographs, chosen for potential difficulty of recognition of abnormalities (Table 2). Each image contained only one abnormality. The chest radiographs were made in either the ambulatory care radiology suite near the emergency room, the regular radiology department, or by portable technique in the medical intensive care unit.

Three magnifications were obtained by adjusting the camera lens; $1 x, 2 x$ and $4 x$. Maximum optical magnification (4x) results in an image equivalent to 16 times the original 
Table 1. Coarse/Detail Lesions

1 Nodule in the pulmonary parenchyma $>3 \mathrm{~cm}$ diame-
ter
1 Pneumonia with consolidation
2 Fractures with displacement/distraction $>1 \mathrm{~cm}$
and/or angulation $>10^{\circ}$
3 Dense calcifications $>2 \mathrm{~cm}$ in one measurement in-
cluding calcified nodes, granulomata, pleural
plaques, and aortic calcification
Air fluid level $>3 \mathrm{~cm}$ in horizontal measurement
CT and ultrasound images with and without lesions
transmitted as 1 -on-1 (each image displayed as a
full screen) display

57

resolution. We compared the relative accuracy of diagnosis using 3 matrix sizes: a $512 \times 480$ matrix, a $1,024 \times 960$ equivalent matrix and a 2,048 $\times 1,920$ equivalent matrix, using the same equipment for each image; only the image magnification was varied.

Acquisition of 4 quadrants of an image with each quadrant magnified up to the full size of the screen, is effectively displayed at 4 times the original resolution, albeit in 4 separate portions, thus the designation "equivalent matrix." While this is not the same as viewing the image on a monitor of higher resolution, it does present a higher information density.

The advantage of using the same equipment is that all other variables such as screen phosphor material, age of equipment, quality of electronics, etc, are excluded from consideration. Any improvement in diagnostic accuracy that results from a larger equivalent matrix size is due only to the increase in information content afforded by the higher resolution and not to other factors.

The digitized images were interpreted without benefit of the original radiographs, by a group of six board-certified or eligible radiologists, each of whom had at least 10 years experience in radiology. Interpretations of the digitized images were compared to those of the original radiographs. The interpretations of the original radiographs was established gold standard; the consensus opinion of three other board-certified radiologists was used.

\section{Light Level Modifications}

A separate experiment was the determination of the effect of modifying the light reaching the vidicon camera from ten

Table 2. Fine Detail Lesions

12 Undisplaced fine fracture lines, no angulation or dis-
traction of fragments
17 Pneumothorax $<20 \%$ of hemithorax transverse diam-
eter
16 Low-density central venous pressure catheters
13 Uncalcified/faintly calcified nodules under $1 \mathrm{~cm}$ diam-
eter
1

images. The light level was modified by selectively masking a low-density region on a chest or bone radiograph prior to image digitization (eg, light shining through the mediastinum and/or subdiaphragmatic region, or around the edges of a small bone film).

\section{RESULTS}

The trial of the 12-on-1, 4-on-1, and 1-on-1 CT and ultrasound images quickly showed that only the 1-on-1 images were readable. (The 12-on-1 transmitted images resulted in a reduction of the original matrix size to less than $128 \times 120$ matrix.) Radiologists would not even call up the 12-on-1 or 4-on-1 images and gave no comment on work sheets regarding them, after their initial extremely negative comments. The 1-on-1 displays, however, amounted to a true $512 \times 480$ image, since the information content on each original CT image was printed at a $512 \times 512$ matrix. The CRT image was an accurate rendition of the original. The same results were obtained with the ultrasound images.

The radiologists did not really like even the 1-on-1 displays on the $512 \times 480$ matrix since viewing all the slices of an examination required that each slice be called up separately, often a tedious task consuming 20 to 30 minutes per examination.

Diagnostic accuracy of the other image interpretations by the radiologists is presented in Table 3, listed according to equivalent matrix size for each area examined. The low diagnostic accuracy of the interpretations of the fine detail (small and/or subtle abnormalities) at the $512 \times$ 480 matrix is in agreement with the "clinically significant discrepancies" found by Kagetsu ${ }^{4}$ who

Table 3. Effect of Matrix Size on True-Positive Fraction (TPF) and False-Positive Fraction (FPF)

\begin{tabular}{|c|c|c|}
\hline & $\begin{array}{l}\text { Average Percent } \\
\text { of Total TPF } \\
\text { (matrix size) }\end{array}$ & $\begin{array}{c}\text { Average Percent } \\
\text { of Total FPF } \\
\text { (matrix size) }\end{array}$ \\
\hline \multicolumn{3}{|c|}{ Total $=59$ fine detail lesions } \\
\hline \multicolumn{3}{|c|}{6 radiologists (354 interpretations) } \\
\hline (a) & 42 & 23 \\
\hline (b) & 73 & 15 \\
\hline (c) & 95 & 2 \\
\hline \multicolumn{3}{|c|}{ Total $=67$ coarse detail lesions } \\
\hline \multicolumn{3}{|c|}{6 radiologists (402 interpretations) } \\
\hline (a) & 82 & 11 \\
\hline (b) & 85 & 7 \\
\hline (c) & 90 & 5 \\
\hline
\end{tabular}

NOTE. Matrix sizes: $a=512 \times 480 ; b=1,024 \times 960 ; c=$ $2.048 \times 1.920$.

* In a few interpretations, more than one lesion was described. 
also used a $512 \times 480$ matrix display. Receiver operating characteristic (ROC) curves were constructed from the six radiologists interpretations of the digitized images at each of the three matrix sizes. (Fig 1)

Light level modification improved the quality in 7 of the 10 images. In one case of the 10 , a nodule just above the diaphragm, the diagnosis was made correctly by all six radiologists but could not be made without masking by any observer.

\section{CONCLUSIONS}

Manufacturers of teleradiology equipment say nothing about the diagnostic accuracy of the equipment to the radiologist, who should realize that not all the information on the original radiographs is present on the transmitted digital image. Any digitized image has lower spatial resolution than the original analog $\mathrm{x}$-ray film image and contains less diagnostic information. A $512 \times 480$ matrix teleradiology system can be easily used for diagnosis of coarse detail images of the type we describe, and for diagnosis of fine detail images with special effort. Diagnosis of fine detail images that would require high spatial resolution can be up to $98 \%$ reliable using equipment with a $512 \times 480$ matrix display if magnification up to $4 \mathrm{x}$ is available before image acquisition, if light level control can be achieved and/or if other steps can be performed such as selective masking.

Other commercial systems offer a $1,024 \times$ 1,024 matrix. A $2,048 \times 2,048$ image display system is available but is not practical for long distance transmission unless considerable compression is used because of the increased time required for transmission.
The decision of which matrix size is required for accurate diagnosis is best made by careful inspection of the $512 \times 480$ digital image and use of the function keys for enhancement. Certainly the transmission time is shorter with a $512 \times 480$ matrix than a $1,024 \times 960$ matrix. Recognition of those areas on the $512 \times 480$ matrix image that may require magnification or light level modification prior to image acquisition may be indicated by abnormal findings on the 512 image or by the patient's history. (It is extremely important to know all pertinent clinical information, before interpreting a $512 \times 480$ matrix image.)

The transmission time of the four quadrants of a radiograph magnified to a $1,024 \times 960(2 x)$ equivalent matrix image plus the entire image at $512 \times 480$, is about 10 minutes at 9,600 baud. Magnification to 2,048 $\times 1,920$ requires another 4 -fold increase since it is further subdivided into 4 parts. The built-in grid overlay serves as an effective localization function to indicate which portions of the image need magnification/ modification. When superimposed on the CRT image, the display shows numbers across the top and letters down the left side. A phone call to the transmitting site need only state which grid squares need to be retransmitted, how much magnification to use, and whether light-level modification is also needed before retransmission.

Another method of using the equipment is conceivable. The original full size image can be acquired and digitized as 4 quadrant images with image quality equivalent to a $1,024 \times 960$ matrix and 16 "subquadrant images" equivalent to $2,048 \times 1,920$ matrix, as well as the $512 \times 480$ matrix of the entire radiograph (acquisition and

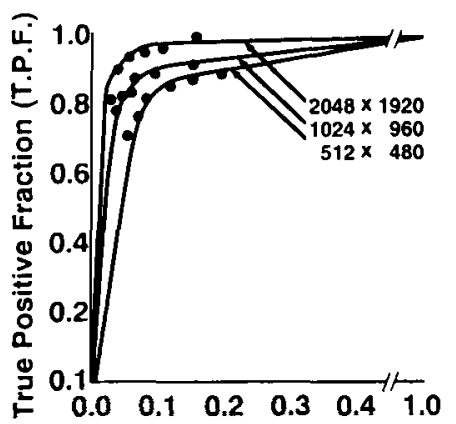

A False Positive Fraction (F.P.F.) Coarse Detail Lesions

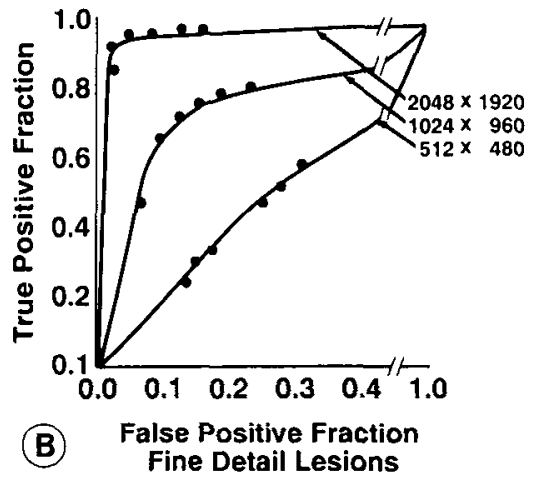

Fig 1. Receiver operating characteristic (ROC) curves for coarse detail (A) and fine detail (B) lesions at each of 3 matrix sizes. 
digitization of each image takes only a few seconds). To save time, only the $512 \times 480$ matrix size is transmitted first. If the receiving radiologist needs the higher quality images, they may be accessed remotely by networking tech- niques without requiring attendance by personnel at the transmitting site. ${ }^{5}$ Storage capability of the transmitting unit must be increased by $20 \mathrm{x}$. Modern hard disk capacity today easily exceeds the $400 \mathrm{mb}$ minimum necessary.

\section{REFERENCES}

1. Gitlin JN: Teleradiology. Radiol Clin North Am 24:5558,1986

2. Harrington MB, Miller KD, Kerlin BD: The 1984-285 teleradiology field trial executive summary. The Mitre Corporation, McLean, VA 1985

3. Markivee CR, Chenoweth J, Patel BK, et al: Diagnostic accuracy of a teleradiology image transmission system. MD Comput 6:88-93, 1989

4. Kagetsu NJ, Zulaut DRP, Ablow RC: Clinical trial of digital teleradiology in the practice of emergency room radiology. Radiology 165:551-554, 1987

5. Markivee CR: Networking of microcomputers in the radiology department. Am J Radiol 145:849-853, 1985 